ders die kohlensauren Salze der fixen Alsalien und aus diesem Grunde rieth Herr Professor Panum schon im Herbste des Jahres 1857 Herrn S. den regelmässigen und täglichen Genuss von Selterwasser an. Herr S. folgte diesem Rathe, indem er täglich eine Flasche dieses Wassers zu sich nalum; es stellte sich dabei eine reichlichere Urinabsonderung ein, so dass statt der fräheren mittleren Menge von $1078 \mathrm{Ccm}$. fortan $1400-1500 \mathrm{Ccm}$. täglich entleert wurden. Der entleerte Harn zeigte gewöhnlich eine alkalische Reaction und liess ein sehr geringes sediment fallen. Herr $\mathbf{S}$. hat den Gebrauch des Selterwassers seitdem mit seltenen Unterbrechungen fortgesetz, und ich bezweiffe es durchaus nicht, dass er sich dadurch allein vor häufigeren und gefährlicheren Zufällen bewahrt bat, welche die Gegenwart scbwer löslicher und krystallisirbarer Körper im Harn so leicht herbeiführt, indem diese Körper schon innerhalb der Harnwege aus dem Menstruum auskrystallisiren und Coneremente bilden.

Bemerken will ich noch, dass ich gleich nach der Entdeckung des Cystins in dem Harn des Herm S., dem Rathe Prout's folgend, den Gebrauch der SalpeterSalzsäure verordnete. Dieses Mittel wurde zwei Manate lang fortgebrancht, allein ich habe nicht die geringste Einwirkung desselben auf die Menge der im Urin entstehenden Cystinsedimente wahrnebmen können.

2.

Ein glücklich verlanfener Fall von Tetanus rhenmaticus.

Beobachtet von Dr. Fr. Asschenfeldt

in Maroim, Provioz Sergipe, Prasilien.

Der Mulatte Sabino Jozé Mauricio, ungefähr 30 Jahre alt, von muskulösem Körperbau, war während einer längeren Flussreise verschiedenen Regengüssen ausgesetzt gewesen, ohne. seine Kleider wechseln zu können. In Folge dessen ward derselbe am 26 . Juli 1862 von einem Tetanus und Trismus rheumaticus befallen.

A $m$ 29. Jali liess mich der Kranke rufen. Ich fand ihn in einem rollkommen tetanischen Zustande; Trismus; Nacken, Extremitäten steif; Bauchmuskeln hart und contrahirt. Mit Hülfe zweier Leute, welche das Hinterbaupt fassten, liess sich der Patient aufrichten, wie ein Brett. — Ganz besonders quälte vollkommene Urinverhaltung; die. Blase war aufgetrieben und schmerzbaft gegen Druck. Der Puls irritirt, ungefähr 100 Sclläge und voll. Stublgang seit Beginn der Krankheit picht erfolgt. - Elektrische Schläge durch das Rückenmark, welche der Kranke als "facadas“, Messerstiche, bezeichnete und die von 2 bis 5 Minuten sich einstellend denselben zu heftigem Schreien zwangen. - Perceptionsvermögen ungestört.

Der eingefïhrte Catheter drang mit Leichtigkeit in die Blase und entleerte eine Menge Urin, worauf Patient schon einige Erleichterung fühlte. 
Ich nahm die Krankheit für eine Myelitis und verordnete: locale Blutentziehungen zu beiden Seiten des Rückgrats, Einreibungen von Ung. hydr. ciner. in Genick und Unterkiefer, und innerlich: Calomel mit Opium.

Gleichzeitig ordnete ich ein Clystier an, bereitet aus Inf. fol. Nicotian. (Gr. sv p.) Unc. iv mit 0l. Ricin. Unc. ij.

Den 30. Juli besuchte ich den Kranken wiederum. Zustand vollkommen derselbe. Ausserdem hatte Patient das verordnete Clystier irrthümlicher Weise getrunken, d. h. durch eine Röhre eingeschlïrft. Es waren in Folge dessen mehr- fache Stuhlentleerungen eingetreten, jedoch ohne Erbrechen, obne Narcose oder sonstige üble Zufälle. - Jetat verordnet: 0 p. pur. Gr. $\beta$ 3stündl. allmälig steigend bis auf Gr. j $\beta$. (Grössere Dosen zu verschreiben, nahm ich Anstand, da, nach meiner Erfahrung, die Farbigen im Allgemeinen ausserordentlich stark auf Narcotica reagiren.) Der Urin abgelassen durch den Cathetér.

Den 31. Juli, Patient hatte etwas geschlafen und fühlte sich in Folge dessen etwas besser, namentlich traten die elektrischen Schläge nach dem- Laufe des Rückenmarkes in etwas längeren Intervallen auf. Urinverhaltung dieselbe und deshalb Application des Catheters.

Die Entfernung, in welcher der Kranke von mir wohnte, verbunden mit den fast unpassirbaren Wegen, machten es mir unmöglich, ibn fernerbin täglich zu besuchen. Deshalb nnterwies ich einen mit dem Patienten in derselben Hütte wohnenden Mulatten in der Einführung des Catheters und anempfabl inm wenigstens 2 Mal während 24 Stunden den Urin zu entfernen. Mit den Opiumpulvern sollte fortgefahren, sowie hin und wieder ein Laxans aus 0l. Ricini und Clystiere aus Inf. fol. Nicotian. (Scr. $\mathrm{j}$ anf Unc. iv) mit 0l. Ricini interponirt werden.

Den 5. Áugust sah ich den Kranken. Es hatte sich mit dem Urin etwas Blut und viel Schleim entleert, wahrscheinlich in Folge ungeschickter Application des Catheters. Im Uebrigen war ein bedeutender Nachlass aller tetanischen Erscheinungen aufgetreten; der Kranke flectirte schon etwas Arme und Beine, der Leib war weicher, der Puls kaum noch etwas alterirt, aber reichliche Schweisse über den ganzen Körper, wenn gleich die Opiumgaben nur bisweilen leichten und kurzen Schlaf hervorgerufen hatten. - -0 effnung war eingetreten, theils freiwillig, theils auf die genannten Clystiere. - Trismus und vollkommene Retentio urinae bestanden fort. - Nach, wie vor der Kranke bei vollster Besinnung.

Volksmittel gegen Tetanus ist in Brasilien ein Syrup, bereitet aus dem Infus des Tabacks. --- Dieses, sowie das neulich ohne weiteren Nachtbeil verschluckte Clystier, veranlassten mich mit dem Opium auszusetzen und Hb. Nicotianae innerlich zu versuchen. Ich verordnete Hec. Fol. Nicotian. Dr. $\beta$, Cort. Cinamom. Dr. ij inf. Aq. ferr. s. q. ad Col. Unc. iv, Syr. simpl. Unc. j. HIDS. 2stündl. 1 Esslöffel.

Den 6. August. Der Kranke hatte nur 2 Esslöffel von vorstehender Arznei genommen, dann aber aufgehört, weil er bebauptete, in Folge dessen seien die elektrischen Schläge durch das Rückenmark mit grösserer Häufigkeit und Heftigkeit, sowie auch die Steifheit der Glieder zu ihrer früheren Höhe zurückgekehrt.

Obgleich ich diesen Rïickfall der nämentlich während der verflossenen Nacht herrschenden, für hiesige Breite (die Provinz Sergipe liegt zwischen $10^{\circ} 30^{\prime}$ und 
$11^{\circ} 32^{\prime}$ S. B.) sehr rauhen und regnigten Witterung, besonders dem starken Sïdwind, gegen welchen die selr zugige und baufällige Wohnung des Kranken keinen Schutz gewährte, zuschrieh, liess ich doch den Patienten wieder seine Opiumpulver nehmen, um die er selbst dringend bat. - Ausserdem verordnete ich viel Wassertrinken, aher nebenher ein stärkendes und näbrendes Regimen: Fleischbrïhe, weichgekochte Eier, Kaffee, guten Wein, Clystiere von Bouillon mit Ei etc., leider gestatteten nur die pecuniären Verhältnisse nicht dem Kranken allen diesen. Vorschriften nachzukommen.

Den 7. August. Zustand dersclbe, allein der entleerte Urin wieder klar.

Den 11. August. Der Kranke fand sich bedeutend erleichtert, namentlich traten die schmerzhaften Schlage durch das Rückgrat yiel seltener auf, Beine und Arme bewegte er leidlich in jeder Richtung, Leih ziemlien weich, Schweisse dauerten fort. Trismus aber, sowie vollhommene Urinverhaltung unverändert, deshalb musste der Catheter, nach wie vor, 2 bis 3 mal und oft häufiger innerhalb 24 Stunden eingeführt werden. - Damit die Umgebung des Kranken nicht zu freigebig mit der Darreichung der Opiumpulver, nach denen der Patient stets verlangte, za Werke ginge, verordnete ich für die folgenden Tage: des Morgens 9 Uhr Gr. j 0p. pur. und Ahends 9 Ubr ein Clystier, bereitet aus Eigelh, mit 39 Tropfen Laud. liq. Sydenham. - Von 3 zu 3 Tagen oder seltener, je nach dem Verhalten der Oeffoung, sollte ein Tag mit diesen Opiumgaben ausgesetzt und stalt dessen ein Laxans aus 0l. Ricin. Unc. ij gegeben werden. Im Uebrigen dasselbe diätetische - Regimen und fortwäbrende Application des Catheters.

Den 25. A ugust besuchte ich den Kranken und fand ibn von allen tetanischen Erscheinungen befreit, laum, dass er noch ein geringes Hinderniss beim Oeffnen und Schliessen des Kiefers verspürte. Er schlief ohne Opium, rauchte, ass mit gutem Appetit und hatte regelmässigen StuhIgang, welche Besserung, nach des Patienten Aussage, seit dem 22sten sich eingestellt. - Bei alledem bestand aber die Harnverhaltung in volkommenem Grade fort, täglich war die mehrmalige Application des Catheters nöthig. - Um diese zu beseitigen batte dem Patienten eine Indierin gerathen eine Abkochang der Haut des Jacaré (Crocodilus Sclerops) zu trinken, indessen wollte derselbe sich nicht dazu verstehen, obne mich consultirt zu haben. Auf die deshalb an mich gerichtete Anfrage allerdings um die Antwort etwas verlegen, allein mich erinnernd, dass die Neger dem Genusse des Fleisches des Kaiman diaphoretische Kräfte zuschreiben, sagte ich dem Kranken, er möchte es nur versuchen.

Den 26. A ugust nabm Patient das erwähnte Mittel und das Resultat davon war in der That evident - innerhalb 5 Minuten nach getrunkener Brïhe urinirte er obne irgend welches Hinderniss, und war bis zum 12. September, an welchem Tage iç den Kranken zuletzt gesehen, kein Blasenleiden irgend einer Art aufgetreten - aer Patient also als rollkommen genesen zu betrachten.

Anmerkung. Die Art, wie die Indianer diese Abkochung bereiten, ist folgende: ein Stück Haut des łacaré, frisch oder getrocknet, von der Länge eines Fingers und ungefähr $\frac{3}{4}$ Zoll Breite, wird mit $1 \frac{1}{2}$ Tassen Wasser üher einem gelinden Feuer 12 Stunden lang gekocht, bis etwa $\frac{1}{2}$ Tasse Flüssigkeit zurückbleibt; 
diese Brühe wird dann a u f einmal getrunken. - Trinkt man hănfiger oder eine stärkere Abkachung, so soll, nach Aussage der Eingebornen, sehr hartnäckige Enuresis die Folge davon sein.

Maroim, den 16. September 1862,

3.

\section{Pathologische Mauserung der Horngebilde in epidemischer Verbreitung. Febris desquamatoria typhodes.}

Von Dr. J. Frank, Kreisphysikus in Züllichau.

Jeder praktische Arzt weiss es, wie manche dunkje Krankheitsvorgänge sich unter der bequemen Bezeichnung: "Nervenfieber ", "Typhoidfieber" verbergen,- und eben nur behandelt werden, otne dass man sich, zur eigenen wissenschaftlichen Beruhigung, über das wahre Wesen derselben Rechenschaft zu geben vermöchte. Eine glïckliche Combination von zufälligen Wahrnehmungen am Krankenbette und Schlussfolgerungen ist oft nur im Stande, die dicltesten Nebel zu lichten, und die gezogenen Resultate der mit Mikroskop und Scalpell ausgerüsteten exacten Forschung, welche dem kleinstädtischen Arzte leiter nicht immer möglich ist, zur Bestătigung zu üherlassen. Von diesem Gesichtspunkte ausgehend wolle der geneigte Leser in folgender Darstellung einer beschränkteren Epidemie im hiesigen Kreise das Resultat einer sorgfältigen und vorurtheilslosen Beobachlung erkennen, und mit Nachsicht beurtbeilen.

Frä̈nlein M. St., 13 J̦ahre alt, von Jymphatischer Conslitution, hatte seit einiger Zeit über schwere Beweglichkeit der Glieder, rasche Ermüdung, Dyspepsie und grosse Gemäthsverstimmung geklagt, welche Erscheinungen, da Patientin im Verhältniss zu ihrem Alter gross und von kräftigem Gliederbau war, auf die sich entwickelnde Menstruation geschoben wurde. - Im Juli v. J. hatte die Mattigkeit einen solchen Grad erreicht, dass sie zu Bette bleiben musste, und zugleich hatten sich gănzliche Appetitlosigkeit, gelblich belegte Zunge und durchfallartige Stuhlgänge eingestell, welchen mässige Auftreibung des Unterleibes, Kollern und grosse Empfindlichkeit der. Ileocoecalgegend, bei geringem Druck, entsprachen. Der Puls war dabei selır erregt, mässig voll und weich, das Gesicht geröthet, das Fieber remittirend, mit abendlichen Exacerbationen. - Exanthem fand sich nirgends vor, anch Leber und Milz zeigten sich weder vergrössert noch scburzhaft. - Schon nach 3 Tagen fing die Kranke an $z \mathfrak{u}$ deliriren, die Zunge wurde roth, lackirt; am sechsten Tage stellten sich bei grosser Hinfälligkeit, so dass Patientin nicht im Stande war, den schmerzenden Kopf zu erheben, duńkler Belag auf der Zunge und am Zabnfleisch ein, Köthe und Hitze des lioptes wurden grösser, die Delirien andauernder, mit Muskelzuclsungen verbunden. Abends Ausbruch duftenden Schweisses mit nachfolgender Erleichterung. - Vom siebenten Tage an verbreitete sicb die Empfindichkeit über den ganzen Unterleib, welcher sich stechend heiss anfühlte, 